\title{
Synthesis and Antimicrobial Evaluation of Novel Carbazole Based $\beta$-diketones and its Pyrazole Derivatives
}

\author{
Vijay A. Kadnor, ${ }^{1}$ Ganesh R. Mhaske,${ }^{2}$ Sharad N. Shelke ${ }^{2, *}$
}

\author{
1 Department of Chemistry, A. C. S. College Satral, Rahuri, Ahmednagar, MS 413711, India \\ 2 Department of Chemistry, S. S. G. M. College Kopargaon, Ahmednagar, MS 423601, India \\ * Corresponding author's e-mail address: snshelke@yahoo.co.in
}

RECEIVED: April 12, 2018 * REVISED: August 20, 2018 * ACCEPTED: October 3, 2018

\begin{abstract}
Novel 9-ethyl-9H-carbazole-3-carboxylic acid derivatives including ester, $\beta$-diketone and pyrazole were prepared and characterized by FT-IR, ${ }^{1} \mathrm{H} N \mathrm{NMR},{ }^{13} \mathrm{C} N \mathrm{NR}$ and mass spectroscopic techniques. All synthesized compounds evaluated for their in vitro antimicrobial activities against four bacteria (Escherichia coli, Pseudomonas putide, Bacillus subtilis, and Streptococcus lactis) and three fungi (Aspergillus niger, Penicillium sp and Candida albicans). Among the compounds tested, 3a, 3b, 3c, 4a, 4b, 4c, 5a and $5 b$ exhibited pronounced antibacterial activity as compared with standard drug ampicillin. Notably, carbazole based pyrazole derivatives $5 \mathbf{a}$ and $\mathbf{5 b}$ showed potent antifungal activity against C. albicans comparable to reference drug greseofulvin.
\end{abstract}

Keywords: antimicrobial activity, carbazole, $\beta$-diketone, pyrazole.

\section{INTRODUCTION}

D ISTINGUISHABLE interest of synthetic organic chemists have attracted considerable attention to carbazole frame because of its derivatives that can be easily reformed by introducing various functional groups. ${ }^{[1]}$ These distinct characteristics results in the broad potential applications of carbazole-based derivatives as industrially and pharmacologically important products (Figure 1).[2] Many recent literatures have reported that carbazole derivatives exhibit a variety of biological activities such as antimicrobial, ${ }^{[3-5]}$ antiviral, ${ }^{[6]}$ anticancer, ${ }^{[7]}$ anti-inflammatory, ${ }^{[8]}$ antimala-rial, ${ }^{[9]}$ antipsychotic ${ }^{[10]}$ and are used in the treatment of obesity. ${ }^{[11]}$

The carbazole carboxylic acid derivatives are significant intermediate because the carboxylic group is one of the active functional group which display an important role in transformation of biological function, these compounds combining low toxicity with high antitumor activity. ${ }^{[12]}$ Functionalized $\beta$-diketones are clinically important molecules showing antibacterial, $[13]$ antiviral, ${ }^{, 14]}$ insecticidal, ${ }^{, 15]}$ antioxidant, ${ }^{[16]}$ potential prophylactic antitumor ${ }^{[17]}$ and pharmacophore of HIV-1 Integrase (IN) inhibitors. ${ }^{[18]}$ The synthesis of $\beta$-diketones containing carbazole fragment and their complexes have already been reported, whereas $\beta$-diketone containing carbazole fragments still remain unknown, though such $\beta$ diketones should be very important and promising for use in optoelectronic materials. ${ }^{[19]} \beta$-diketones are important intermediates for the synthesis of medicinally important heterocycles such as pyrazole, ${ }^{[20,21]}$ because of their derivatives represent one of the most active classes of compounds and possess a wide spectrum of biological activities. ${ }^{[22-24]}$ Insight the literature, carbazole based pyrazole derivatives possesses potent antibacterial and antifungal activities. ${ }^{[25]}$ In continuation of our studies in synthesizing various biologically active compounds, ${ }^{[26,27]}$ in this study, we have synthesized and characterized the novel carbazole assembled esters, $\beta$-diketones and pyrazoles derivatives from 9-ethyl-9H-carbazole-3-carboxylic acid and evaluated for in vitro antibacterial and antifungal activities. 
<smiles>CCn1c2ccccc2c2cc(NC(=O)c3ccccc3Cl)ccc21</smiles>

3-Benzamidocarbazole<smiles>CCn1c2ccccc2c2cc(S(=O)(=O)Nc3cc(OC)c(OC)c(OC)c3)ccc21</smiles>

Carbazole sulphonamides<smiles>CC(C(=O)O)c1ccc2c(c1)[nH]c1ccc(Cl)cc12</smiles>

Carprofen

Figure 1. Biologically active synthetic carbazole derivatives.

\section{RESULTS AND DISCUSSION}

\section{Chemistry}

In view of the emerging biological importance of carbazole, it was of interest to synthesize some carbazole assembled esters, $\beta$-diketones and pyrazoles derivatives on the hope of obtaining more antimicrobial agents. Thus, starting compound, 9-ethyl-9H-carbazole-3-carboxylic acid 2 was prepared from oxidation of 9-ethyl-9Hcarbazole-3-carbaldehyde by literature method. ${ }^{[28]}$ In the present work 2-hydroxy acetophenones 1 were treated with 9-ethyl-9H-carbazole-3-carboxylic acid $\mathbf{2}$ in the presence of phosphorous oxychloride and pyridine to afford the corresponding esters 3(a-e). Carbazole esters 3(a-e) treated with strong base like potassium hydroxide in the presence of pyridine bring an intramolecular Claisen condensation as per Baker-Venkataraman (Bk-Vk) transformation, ${ }^{[29-30]}$ resulting in 1-(9-ethyl-9H-carbazol3-yl)-3-(2-hydroxyphenyl) propane-1,3-dione 4(a-e). In the next step, cyclization of the $\beta$-diketones using hydrazine hydrate in ethanol at reflux temperature gave pyrazoles 5(a-e) as shown in Scheme 1. The BakerVenkataraman transformation proceeds via the formation of an enolate 3a followed by an intramolecular acyl transfer Scheme 2.

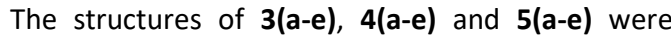
confirmed by FT-IR, ${ }^{1} \mathrm{H}$ and ${ }^{13} \mathrm{C}$ NMR, and mass spectra. For example, the infrared spectra of $\mathbf{3}(\mathbf{a}-\mathbf{e})$ shows an intense absorption band at around $1735 \mathrm{~cm}^{-1}$ for $-\mathrm{O}-\mathrm{CO}$ group occurs at higher frequencies than that of normal ketones because force constant of the carbonyl bond is increased by the electron attracting nature of adjacent oxygen atom and the ${ }^{1} \mathrm{HNMR}$ spectrum of $\mathbf{3 a}$ contained characteristic singlet at $\delta 2.55 \mathrm{ppm}$ for $\mathrm{CO}-\mathrm{CH}_{3}$ which confirmed the esterification of 9-ethyl-9H-carbazole-3-carboxylic acid 2, aromatic protons resonated in the region $\delta 7.33-9.01 \mathrm{ppm}$. In the ${ }^{13} \mathrm{C}$ NMR spectra of 3 (a-e) showed aromatic carbon signals in the region of $\delta 108.47-145.59 \mathrm{ppm}$, whereas conjugated carbonyl ester appeared at $\delta 164.59 \mathrm{ppm}$ and carbonyl carbon at $\delta 195.67 \mathrm{ppm}$. The mass spectrum of 3a displayed a molecular ion peaks at $\mathrm{m} / \mathrm{z} 426[\mathrm{~m}+1]$, $427[\mathrm{~m}+2]$ and $429[\mathrm{~m}+4]$ confirmed the compound $3 a$ contained two chlorine atoms.

The infrared spectra of $\mathbf{4}(\mathrm{a}-\mathrm{e})$ shows a strong and characteristic band for 1,3-diketone linkage at 1677-1590 $\mathrm{cm}^{-1}$ and $2979 \mathrm{~cm}^{-1}$ for $-\mathrm{OH}$ stretching. The representative ${ }^{1} \mathrm{HNMR}$ spectrum of $\mathbf{4} \mathbf{b}$ shows disappearance of a singlet at around $\delta 2.55 \mathrm{ppm}$ (corresponding to $\mathrm{CO}-\mathrm{CH}_{3}$ ) but it displayed two sharp singlets due to two protons at $\delta 16.27$ ppm and $\delta 12.48 \mathrm{ppm}$, which confirm the presence of enolic proton (since enol form in $\beta$-diketone is more stable) and phenolic $-\mathrm{OH}$ adjacent to the carbonyl group respectively. ${ }^{13} \mathrm{C}$ NMR spectra showed a singlet at $\delta 203.07 \mathrm{ppm}$ due to ketonic carbon and at $\delta 168.47 \mathrm{ppm}$ due to enolic carbon confirming the keto-enol tautomerism in $\beta$-diketone $\mathbf{4 b}$. The negative test for ester, the presence of characteristic ${ }^{1} \mathrm{HNMR}$ and ${ }^{13} \mathrm{C}$ NMR peaks are consistent with the structure $\mathbf{4 b}$ and aromatic carbon signals of compounds $4(\mathrm{a}-\mathrm{e})$ observed in the region of $\delta 109.25-142.53 \mathrm{ppm}$. The mass spectrum of $\mathbf{4} \mathbf{b}$ displayed a molecular ion peak at $m / z$ $392[\mathrm{~m}+1]$. The infrared spectrum of $5 \mathrm{a}$ showed the appearance of absorption band at 3373,3246 and $1455 \mathrm{~cm}^{-1}$ corresponding to $\mathrm{NH}, \mathrm{OH}$ and $\mathrm{C}=\mathrm{N}$ functional group respectively. Also, its ${ }^{1} \mathrm{HNMR}$ spectrum supported its structure, as it revealed the pyrazole ring protons at $\delta 7.26$ and two broad signals at $\delta 12.61$ and $8.80 \mathrm{ppm}$ assignable to $\mathrm{OH}$ and $\mathrm{NH}$ protons, respectively. The ${ }^{13} \mathrm{C}$ NMR spectrum of the compounds $\mathbf{5}(\mathrm{a}-\mathrm{e})$ showed aromatic carbon signals in the region $\delta 109.20-140.25 \mathrm{ppm}$.

\section{Antibacterial and Antifungal Evaluation}

Antimicrobial activity of newly synthesized compounds 3,4 and $\mathbf{5}$ was evaluated against two gram negative (E. coli, $P$. putide), two gram positive (B. subtilis, S. lactis) bacterial strains, and three (A. niger, Penicillium sp, $C$. albicans) fungal strains using Ampicillin and Greseofulvin as a standard drugs respectively. The inhibition zone diameter $(\mathrm{mm})$ and minimal inhibitory concentration (MIC) values of all synthesized compounds were noted in Table 1. Graphical representations Figure 2 and 3, inhibition zone diameter $(\mathrm{mm})$ against a compound number $(\mathbf{3}, \mathbf{4}$ and $\mathbf{5})$, exhibiting moderate to a promising activity against tested 
<smiles>[R]c1cc(C(C)=O)c(O)c([R])c1[R]</smiles><smiles>CCC(N)C(C)N</smiles>

3a, 4a, 5a : $\mathrm{R}^{1}=\mathrm{Cl}, \mathrm{R}^{2}=\mathrm{H}, \mathrm{R}^{3}=\mathrm{Cl}$<smiles>[R]c1cc(-c2cc(-c3ccc4c(c3)c3ccccc3n4CC)[nH][Y9]2([H])[H])c(O)c([R])c1[R]</smiles>

$3 b, 4 b, 5 b: R^{1}=C l, R^{2}=H, R^{3}=H$

3c, 4c, $5 c: R^{1}=B r, R^{2}=H, R^{3}=H$

3d, 4d, 5d : $\mathbf{R}^{1}=\mathrm{CH}_{3}, \mathrm{R}^{2}=\mathrm{H}, \mathrm{R}^{3}=\mathrm{H}$

$3 e, 4 e, 5 e: R^{1}=H, R^{2}=H, R^{3}=H$

Scheme 1 . Synthetic route of target compounds 3,4 and 5 .<smiles>[R]c1cc(C(=O)O)c(OCC(=O)c2ccc3c(c2)c2ccccc2n3CC)c([Hg])c1[R]</smiles><smiles>[R]c1cc(C(=O)CC(=O)c2ccc3c(c2)c2ccccc2n3CC)c([O-])c([R3])c1[R]</smiles>

Scheme 2. Mechanism of the Baker-Venkataraman (Bk-Vk) transformation.

bacterial and fungal strains as compared with standard drugs. It was found that compounds $\mathbf{3}(\mathbf{a}-\mathbf{d}), \mathbf{3 a}, \mathbf{3 b}$ and $\mathbf{3 c}$ gave stronger antibacterial efficacies and broader bioactive spectrum against $S$. lactis, and $B$. subtilis with the MIC 
Table 1. Antimicrobial activities(a) of the synthesized compounds 3, 4 and 5 against pathological organisms expressed as inhibition diameter zones in millimeters $(\mathrm{mm})$ and ${ }^{(b)} \mathrm{MIC}(\mu \mathrm{g} / \mathrm{mL}$, between brackets)

\begin{tabular}{|c|c|c|c|c|c|c|c|}
\hline \multirow{3}{*}{ Compd.no } & \multicolumn{7}{|c|}{ Microorganisms } \\
\hline & \multicolumn{2}{|c|}{ Gram -ve bacteria } & \multicolumn{2}{|c|}{ Gram +ve bacteria } & \multicolumn{3}{|c|}{ Fungi } \\
\hline & $\begin{array}{l}\text { Escherichia } \\
\text { coli }\end{array}$ & $\begin{array}{l}\text { Pseudomonas } \\
\text { putide }\end{array}$ & $\begin{array}{l}\text { Bacillus } \\
\text { subtilis }\end{array}$ & $\begin{array}{l}\text { Streptococcus } \\
\text { lactis }\end{array}$ & $\begin{array}{l}\text { Aspergillus } \\
\text { niger }\end{array}$ & Penicillium $s p$ & $\begin{array}{l}\text { Candida } \\
\text { albicans }\end{array}$ \\
\hline $3 a$ & $14(90)$ & $12(80)$ & $16(40)$ & $20(30)$ & $18(80)$ & $10(80)$ & $11(45)$ \\
\hline $3 b$ & $18(100)$ & $15(90)$ & $17(35)$ & $20(30)$ & $16(100)$ & $10(100)$ & $12(50)$ \\
\hline $3 c$ & $11(90)$ & $14(80)$ & $14(40)$ & $18(40)$ & 19(90) & $11(55)$ & $09(80)$ \\
\hline $3 d$ & $17(100)$ & $18(75)$ & $18(90)$ & $17(80)$ & $17(100)$ & $12(90)$ & $10(95)$ \\
\hline $3 e$ & $14(100)$ & $12(100)$ & $15(90)$ & $18(100)$ & $11(90)$ & $11(100)$ & $08(100)$ \\
\hline $4 a$ & $16(100)$ & $15(75)$ & $14(80)$ & $17(35)$ & $12(90)$ & $12(30)$ & 09(85) \\
\hline $4 b$ & $12(90)$ & $13(65)$ & $12(80)$ & $17(40)$ & $17(80)$ & $11(30)$ & $12(90)$ \\
\hline $4 c$ & $11(100)$ & $13(80)$ & $14(90)$ & $19(45)$ & $13(100)$ & $12(40)$ & $11(80)$ \\
\hline $4 d$ & $12(110)$ & $17(100)$ & $11(100)$ & $16(90)$ & $12(100)$ & $12(55)$ & NA \\
\hline $4 \mathrm{e}$ & $16(100)$ & $14(100)$ & 09(110) & $15(100)$ & $15(110)$ & $11(80)$ & NA \\
\hline $5 a$ & $16(90)$ & $13(45)$ & $18(35)$ & $21(45)$ & $17(95)$ & $10(85)$ & $16(25)$ \\
\hline $5 b$ & $16(90)$ & $16(55)$ & $17(35)$ & $21(50)$ & 19(90) & 10(90) & $15(30)$ \\
\hline $5 c$ & $13(100)$ & $14(90)$ & $16(70)$ & $19(100)$ & $18(85)$ & $11(100)$ & $09(60)$ \\
\hline $5 d$ & $16(120)$ & $18(100)$ & $15(110)$ & $15(90)$ & $17(90)$ & $12(90)$ & $12(100)$ \\
\hline $5 e$ & $14(110)$ & $16(95)$ & $15(110)$ & $16(110)$ & $11(100)$ & $11(100)$ & $11(90)$ \\
\hline Ampicillin & $24(25)$ & $20(25)$ & $19(25)$ & $22(25)$ & ----- & ----- & ----- \\
\hline Greseofulvin & ----- & ----- & ----- & ---- & $24(25)$ & $14(25)$ & $14(25)$ \\
\hline $\begin{array}{l}\text { Control } \\
\text { (1\%DMSO) }\end{array}$ & NA & NA & NA & NA & NA & NA & NA \\
\hline
\end{tabular}

(a) Inhibition zone diameters were measured for stock solutions $(100 \mu \mathrm{g} / \mathrm{mL})$.

(b) Minimal inhibitory concentration (MIC) values. $1 \%$ DMSO was used as control. NA- No activity.

values in the range $(30-40 \mu \mathrm{g} / \mathrm{mL})$ comparable to that of the positive control, also compounds $\mathbf{3 d}$ and $\mathbf{3 e}$ exhibit moderate to good inhibitory activities (75 and $90 \mu \mathrm{g} / \mathrm{mL}$ ) against $P$. putide and $B$. subtilis bacterial strain respectively. Compounds $\mathbf{3 a}, \mathbf{3 b}$ and $\mathbf{3} \mathbf{c}$ showed a broad spectrum of antifungal activities $(45-55 \mu \mathrm{g} / \mathrm{mL})$ against $C$. albicans and Penicillium $s p$ as compared with standard drug greseofulvin. Among $\beta$-diketones 4(a-e), compounds $\mathbf{4 a}, \mathbf{4 b}$ and $\mathbf{4 c}$ showed good inhibition activities $(35-45 \mu \mathrm{g} / \mathrm{mL}$ ) against $S$. lactis bacterial strains, remaining members could be able to prevent the growth of testing bacterial strains comparable

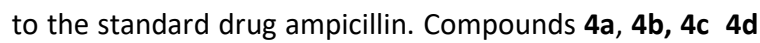
and 4 e displayed significant inhibition activities with a MIC $\geq 30 \mu \mathrm{g} / \mathrm{mL}$ against all tested fungal strains, while compounds $\mathbf{4 d}$ and $\mathbf{4 e}$ are passive for $C$. albicans fungal strain. Carbazole based pyrazoles $\mathbf{5}(\mathbf{a}-\mathbf{e})$, compounds $\mathbf{5 a}$ and $\mathbf{5 b}$ shows remarkable antibacterial activity against tested pathogens namely $S$. lactis, $B$. subtilis and $P$. putide compared to standard drug ampicillin at lowest concentration ranging from $(35-55 \mu \mathrm{g} / \mathrm{mL}$ ) with nearly equipotent of inhibition zone, compounds $5 \mathbf{d}$ and $\mathbf{5 e}$ could not effectively inhibit the growth of all tested bacterial strains. Compounds $\mathbf{5 a}$ and $\mathbf{5 b}$ showed maximum antifungal activities with MIC value ( 25 and $30 \mu \mathrm{g} / \mathrm{mL}$ ) against $C$. albicans as compared with commercial antibiotic greseofulvin. While most of the compounds $\mathbf{3 , 4}$ and $\mathbf{5}$ were not satisfactorily inhibit the growth of $E$. coli bacterial strain as compared with positive control.

\section{CONCLUSIONS}

Novel 9-ethyl-9H-carbazole-3-carboxylic acid derivatives including ester, $\beta$-diketone and pyrazole were prepared investigated for their in vitro antimicrobial activities. Among the synthesized compounds, compounds $\mathbf{3} \mathbf{a}, \mathbf{3} \mathbf{b}, \mathbf{3} \mathbf{c}$, $\mathbf{4 a}, \mathbf{4 b}$ and $\mathbf{4 c}$ showed moderate to promising antimicrobial activities in comparison with standard drug. In addition to compounds $\mathbf{5 a}$ and $\mathbf{5 b}$ were identified as the most potent antibacterial and antifungal agents compared with reference compounds. As structure activity relationship 


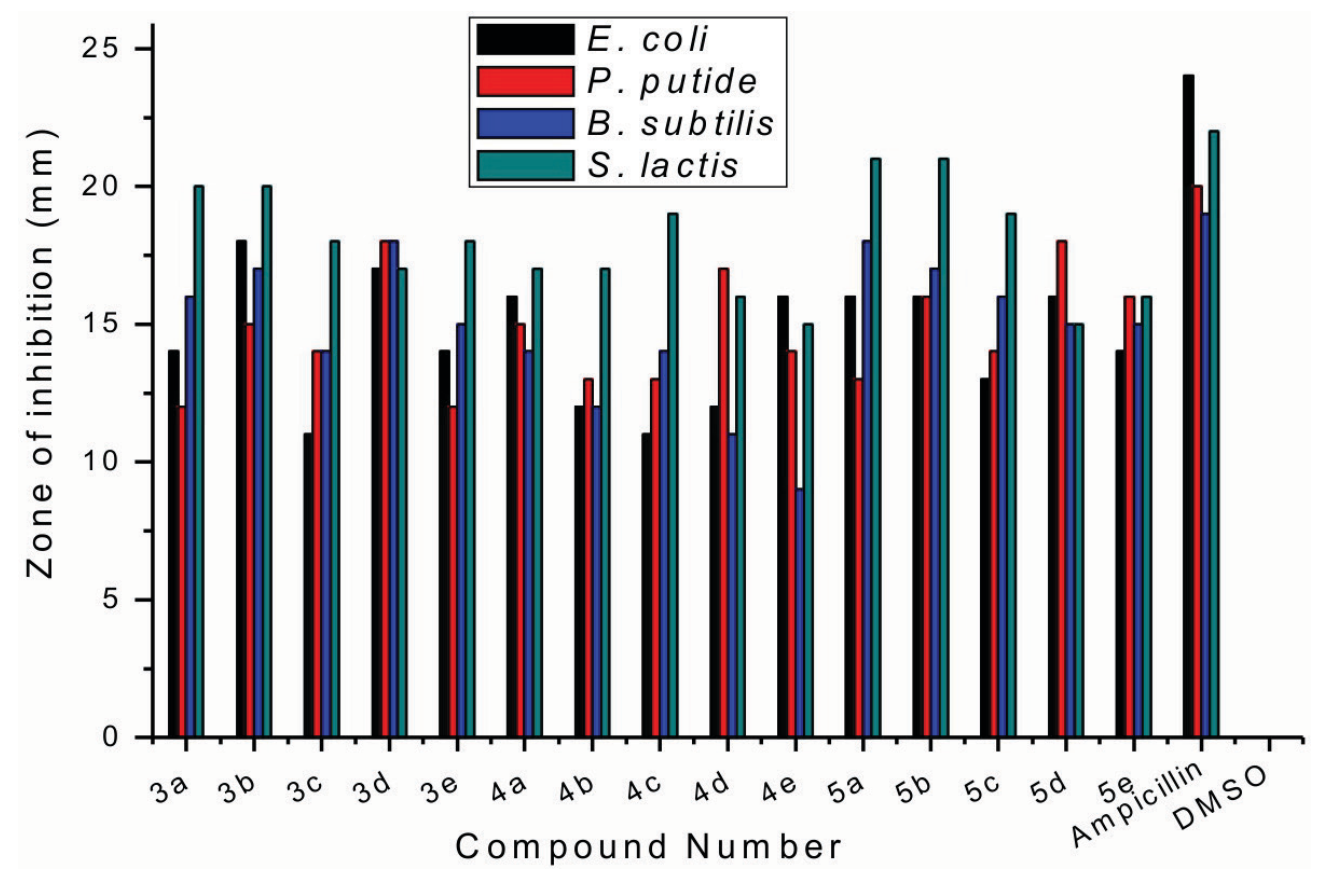

Figure 2. Antibacterial activities of the synthesized compounds 3, 4 and 5.

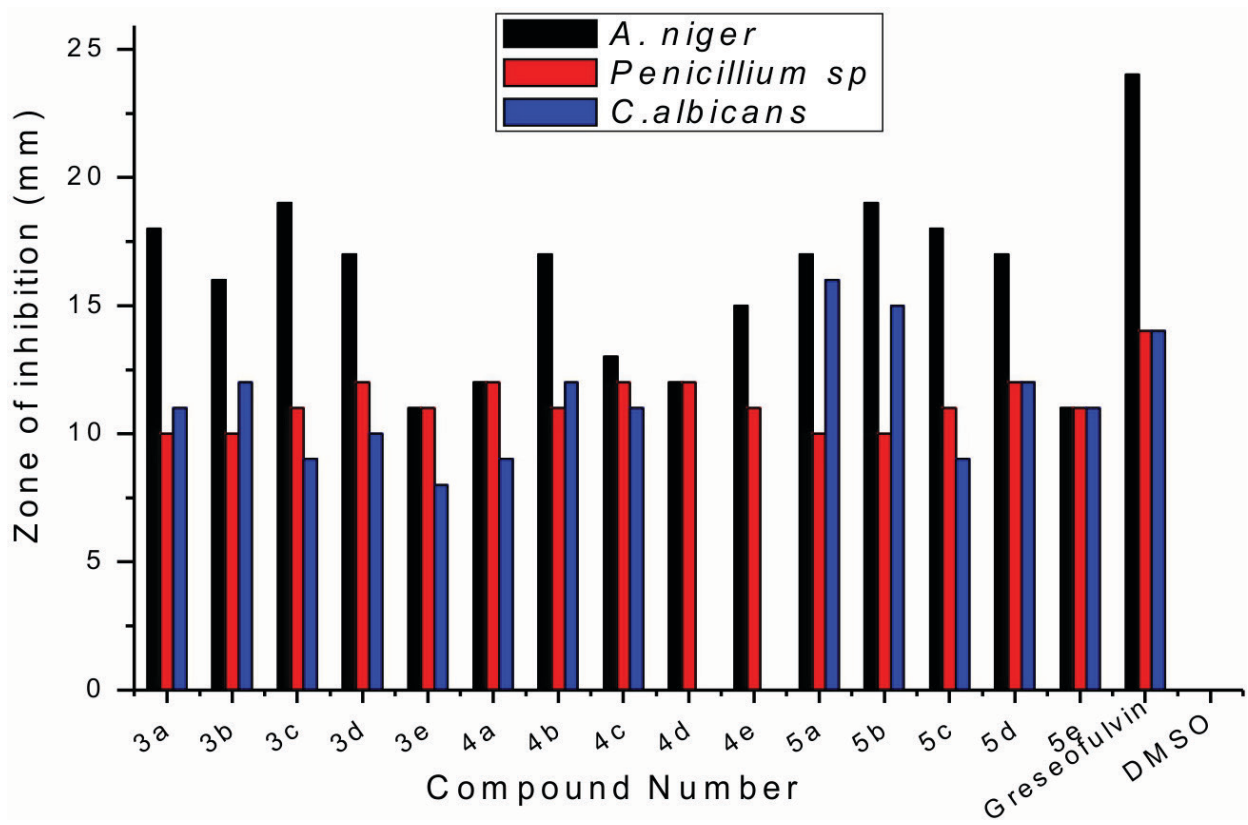

Figure 3. Antifungal activities of the synthesized compounds 3,4 and 5 .

(SAR) study of all compounds were taken into account, it was observed that the introduction of carbazole moiety to $\beta$-diketone, ester and pyrazole derivatives caused enriched activities against most test organisms. The results also suggested that the antimicrobial activities of the carbazole derivatives were distinctly influenced by the aromatic substituents. Compounds 3a, 3b, 3c, 4a, 4b, 4c, 5a, 5b and $5 \mathrm{c}$ with electron withdrawing substituents $(\mathrm{Cl}$ and $\mathrm{Br})$ in the 
phenyl ring were more potent against most of the tested microorganisms than compounds with electron donating ones. Furthermore, compounds $\mathbf{3 e}, \mathbf{4 e}$ and $\mathbf{5 e}$ without substituent in the phenyl ring showed satisfactory activities against all tested bacterial and fungal strains. High potency and promising antimicrobial activity of newly synthesized compounds $\mathbf{3}(\mathrm{a}-\mathrm{e}), \mathbf{4}(\mathrm{a}-\mathrm{e})$ and $\mathbf{5}(\mathrm{a}-\mathrm{e})$ suggest that these compounds could serve as good leads for further optimization and development.

\section{EXPERIMENTAL}

The recorded melting points were determined in an open capillary and are uncorrected. IR spectra were recorded on Perkin Elmer Fourier-transform infrared (FTIR) spectrometer from $\mathrm{KBr}$ pellets. The ${ }^{1} \mathrm{H} N \mathrm{NMR}$ and ${ }^{13} \mathrm{C}$ NMR spectra were recorded on Bruker Avance II (400 MHz) and Bruker (125 MHz) spectrometer respectively, using TMS as internal standard. Mass spectra were recorded on a Waters, Q-TOF micromass, while HRMS were scanned on Bruker impact HD (ESI-Q-TOF) spectrophotometer. The thin layer chromatography (TLC) was carried out on precoated silica gel aluminum plates to check compound purity. The substituted 2-hydroxyacetophenones are commercially available.

\section{In Vitro Antimicrobial Assay}

The antimicrobial activity was evaluated by the agar well diffusion method. The activity was determined by measuring the diameter of inhibition zone (in $\mathrm{mm}$ ). The samples of the tested compound concentrations $(50 \mu \mathrm{L}, 1 \mathrm{mg} / \mathrm{mL})$ were loaded into wells on the plates. All solutions were prepared in DMSO, and pure DMSO was loaded as a control. The plates were incubated at 37 ㄷ for 1-5 days and then were examined for the formation of inhibition zone. Each inhibition zone was measured three times to get an average value. The test was performed three times for each bacterium culture. ${ }^{[31]}$

\section{Minimal Inhibitory Concentration (MIC) Measurement}

The potato dextrose broths and microorganisms susceptibility tests in nutrient media were used for the determination of MIC. The tested compounds stock $1000 \mu \mathrm{g} / \mathrm{mL}$ solutions, Ampicillin and Greseofulvin were prepared in DMSO followed by dilutions to $250-25 \mu \mathrm{g} / \mathrm{mL}$ concentrations. Inoculated microorganism suspensions were incubated at $37^{\circ} \mathrm{C}$ for $1-5$ days for MIC determination. [31].

\section{General Procedure for Esterification of Compounds 3(a-e)}

A mixture of compound 1 (1.36 g, $10 \mathrm{mmol}$ ) and 9-ethyl-9Hcarbazole-3-carboxylic acid $\mathbf{2}(2.3 \mathrm{~g}, 10 \mathrm{mmol})$ was dissolved in dry pyridine $(10 \mathrm{~mL})$. Cooled the flask in an ice bath and phosphorousoxychloride $(1.53 \mathrm{~g}, 10 \mathrm{mmol}$ ) was added dropwise with constant stirring while maintain the temperature between $0-10{ }^{\circ} \mathrm{C}$. After complete addition of phosphorousoxychloride, the reaction mixture was kept overnight at room temperature, then poured over crushed ice and acidified using cold dilute $\mathrm{HCl}$. The off white solid product obtained was filtered and washed with cold dill. $\mathrm{NaHCO}_{3}$ solution followed by washing with cold water. Crude product was dried and recrystallized from ethanol to obtain the desired product in pure form $\mathbf{3}(\mathbf{a}-\mathbf{e})$, which gave a positive test for ester.

2-acetyl-4, 6-dichlorophenyl 9-ethyl-9H-carbazole-3-carboxylate (3a) Off white solid; Yield (73\%); $R_{\mathrm{f}}=0.44$ (6\% ethylacetate in $n$-hexane); m.p. $98-99{ }^{\circ} \mathrm{C}$; IR (KBr) $\tilde{v}_{\text {max }} /$ $\mathrm{cm}^{-1}$ : $1130(\mathrm{C}-\mathrm{Cl}), 1199(\mathrm{C}-\mathrm{O}), 1697(\mathrm{C}=\mathrm{O}), 1731$ (ester $\mathrm{C}=\mathrm{O}) ;{ }^{1} \mathrm{H} \mathrm{NMR}\left(\mathrm{CDCl}_{3}\right) \delta / \mathrm{ppm}: 1.48\left(\mathrm{t}, 3 \mathrm{H}, \mathrm{CH}_{3}\right), 2.55(\mathrm{~s}, 3 \mathrm{H}$, $\left.\mathrm{COCH}_{3}\right), 4.43\left(\mathrm{q}, 2 \mathrm{H}, \mathrm{N}-\mathrm{CH}_{2}\right), 7.33(\mathrm{~m}, 1 \mathrm{H}, \mathrm{ArH}), 7.51(\mathrm{~m}, 3 \mathrm{H}$, ArH), $7.66(\mathrm{~d}, J=2.5 \mathrm{~Hz}, 1 \mathrm{H}, \mathrm{ArH}), 7.74(\mathrm{~d}, J=2.5 \mathrm{~Hz}, 1 \mathrm{H}$, ArH), $8.18(\mathrm{~d}, J=7.7 \mathrm{~Hz}, 1 \mathrm{H}, \mathrm{ArH}), 8.35$ (dd, $J=1.6 \& J=7.0$ $\mathrm{Hz}, 1 \mathrm{H}, \mathrm{ArH}), 9.01(\mathrm{~d}, J=1.5 \mathrm{~Hz}, 1 \mathrm{H}, \mathrm{ArH}) ;{ }^{13} \mathrm{C} \mathrm{NMR}\left(\mathrm{CDCl}_{3}\right)$ $\delta / \mathrm{ppm}: 13.84,30.04,37.95,108.47,109.10,118.20$, $120.32,120.93,122.96,123.13,124.23,124.37,126.78$, $128.16,128.39,130.21,131.90,133.33,134.67,140.69$, $143.42,145.18,164.59,195.67$; $\mathrm{MS}(\mathrm{m} / \mathrm{z}): 426(\mathrm{M}+\mathrm{H})^{+}$. HRMS (ESI): calculated for $\mathrm{C}_{23} \mathrm{H}_{17} \mathrm{Cl}_{2} \mathrm{NNaO}_{3} \quad(\mathrm{M}+\mathrm{Na})$ 448.047769 , found 448.0480 .

2-acetyl-4-chlorophenyl 9-ethyl-9H-carbazole-3-carboxylate (3b) Off white solid; Yield (70\%); $R_{f}=0.49(6 \%$ ethylacetate in $n$-hexane); m.p. $112-113{ }^{\circ} \mathrm{C}$; IR (KBr) $\tilde{v}_{\text {max }} / \mathrm{cm}^{-1}$ : $1131(\mathrm{C}-\mathrm{Cl}), 1200$ (C-O), 1687 (C=O), 1733 (ester $\mathrm{C}=0) ;{ }^{1} \mathrm{H} \mathrm{NMR}\left(\mathrm{CDCl}_{3}\right) \delta / \mathrm{ppm}: 1.49\left(\mathrm{t}, 3 \mathrm{H}, \mathrm{CH}_{3}\right), 2.56(\mathrm{~s}, 3 \mathrm{H}$, $\left.\mathrm{COCH}_{3}\right), 4.45\left(\mathrm{q}, 2 \mathrm{H}, \mathrm{N}-\mathrm{CH}_{2}\right), 7.18-7.26(\mathrm{~m}, 2 \mathrm{H}, \mathrm{ArH})$, 7.33-7.66 (m, 4H, ArH), 7.77-8.16 (m, 2H, ArH), 8.31-8.99 $(\mathrm{m}, 2 \mathrm{H}, \mathrm{ArH}) ;{ }^{13} \mathrm{C} \mathrm{NMR}\left(\mathrm{CDCl}_{3}\right) \delta / \mathrm{ppm}: 13.72,30.28,37.53$, $108.16,109.05,118.16,119.80,120.03,120.90,122.63$, $123.07,124.49,124.94,126.59,128.45,128.90,130.10$, $131.96,133.82,134.27,140.52,143.15,145.23,174.46$, 192.74; MS (m/z): $392(\mathrm{M}+\mathrm{H})^{+}$. HRMS (ESI): calculated for $\mathrm{C}_{23} \mathrm{H}_{18} \mathrm{ClNNaO}_{3}(\mathrm{M}+\mathrm{Na})$ 414.086742, found 414.086845 .

2-acetyl-4-bromophenyl 9-ethyl-9H-carbazole-3-carboxylate (3c) Off white solid; Yield (67\%); $R_{f}=0.42(6 \%$ ethylacetate in n-hexane); m.p. $153-154{ }^{\circ} \mathrm{C}$; IR (KBr) $\tilde{V}_{\max } / \mathrm{cm}^{-1}: 1033$ (C-Br), 1239 (C-O), 1697 (C=O), 1732 (ester $\mathrm{C}=\mathrm{O}) ;{ }^{1} \mathrm{H} \mathrm{NMR}\left(\mathrm{CDCl}_{3}\right) \delta / \mathrm{ppm}: 1.47\left(\mathrm{t}, 3 \mathrm{H}, \mathrm{CH}_{3}\right), 2.62(\mathrm{~s}, 3 \mathrm{H}$, $\left.\mathrm{COCH}_{3}\right), 4.42\left(\mathrm{q}, 2 \mathrm{H}, \mathrm{N}-\mathrm{CH}_{2}\right), 7.24-7.26(\mathrm{~m}, 2 \mathrm{H}, \mathrm{ArH}), 7.31-$ $7.36(\mathrm{~m}, 2 \mathrm{H}, \mathrm{ArH}), 7.44-7.55(\mathrm{~m}, 3 \mathrm{H}, \mathrm{ArH}), 8.18-8.28(\mathrm{~m}, 2 \mathrm{H}$, ArH). $8.93(\mathrm{~s}, 1 \mathrm{H}, \mathrm{ArH}) ;{ }^{13} \mathrm{C} \mathrm{NMR}\left(\mathrm{CDCl}_{3}\right) \delta / \mathrm{ppm}: 13.97$, $33.09,37.76,106.98,107.73,108.84,110.13,119.35$, $119.57,120.25,120.45,120.47,122.64,123.08,126.37$, $127.48,133.09,138.90,140.76,143.37,155.46,158.07$, 172.01, 191.85; MS $(\mathrm{m} / \mathrm{z}): 436(\mathrm{M}+\mathrm{H})^{+}$. HRMS(ESI): calculated for $\mathrm{C}_{23} \mathrm{H}_{18} \mathrm{BrNNaO}_{3}(\mathrm{M}+\mathrm{Na})$ 458.047012, found 458.047019 . 
2-acetyl-4-methylphenyl 9-ethyl-9H-carbazole-3-carboxylate (3d) Off white solid; Yield (70\%); $R_{f}=0.52(6 \%$ ethylacetate in n-hexane); m.p. 138-139 ${ }^{\circ} \mathrm{C}$; IR (KBr) $\tilde{V}_{\max } / \mathrm{cm}^{-1}: 1033$ (C-O), $1692(\mathrm{C}=\mathrm{O}), 1731$ (ester $\left.\mathrm{C}=\mathrm{O}\right) ;{ }^{1} \mathrm{H}$ $\operatorname{NMR}\left(\mathrm{CDCl}_{3}\right) \delta / \mathrm{ppm}: 1.49\left(\mathrm{t}, 3 \mathrm{H}, \mathrm{CH}_{3}\right), 2.43\left(\mathrm{~s}, 3 \mathrm{H}, \mathrm{Ar}-\mathrm{CH}_{3}\right)$, $2.55\left(\mathrm{~s}, 3 \mathrm{H}, \mathrm{COCH}_{3}\right), 4.44\left(\mathrm{q}, 2 \mathrm{H}, \mathrm{N}-\mathrm{CH}_{2}\right), 7.17-7.26(\mathrm{~m}, 2 \mathrm{H}$, ArH), 7.32-7.40 (m, 2H, ArH), 7.49-7.67 (m, 2H, ArH), 7.79-7.82 (m, 2H, ArH), $8.16(\mathrm{~m}, 1 \mathrm{H}, \mathrm{ArH}), 8.31(\mathrm{~m}, 1 \mathrm{H}$, $\mathrm{ArH}) ;{ }^{13} \mathrm{C} \mathrm{NMR}\left(\mathrm{CDCl}_{3}\right) \delta / \mathrm{ppm}: 13.51,20.98,29.85,37.98$, $107.94,108.62,119.35,119.80,120.48,123.06,123.53$, $123.97,124.51,126.07,127.97,130.31,130.99,133.53$, 135.39, 140.54, 143.16, 147.33, 165.97, 198.35; MS (m/z): $372(\mathrm{M}+\mathrm{H})^{+}$. HRMS(ESI): calculated for $\mathrm{C}_{24} \mathrm{H}_{21} \mathrm{NNaO}_{3}$ $(\mathrm{M}+\mathrm{Na}$ ) 394.012145, found 394.012150.

2-acetylphenyl 9-ethyl-9H-carbazole-3-carboxylate (3e) Off white solid; Yield (69\%); $R_{\mathrm{f}}=0.48$ (6\% ethylacetate in $\mathrm{n}$ hexane); m.p. $198-199^{\circ} \mathrm{C} ; \mathrm{IR}(\mathrm{KBr}) \tilde{v}_{\max } / \mathrm{cm}^{-1}: 1124$ (C-O), 1626 $(\mathrm{C}=\mathrm{O}), 1706$ (ester $\mathrm{C}=\mathrm{O})$; ${ }^{1} \mathrm{H} \mathrm{NMR}\left(\mathrm{CDCl}_{3}\right) \delta / \mathrm{ppm}$ : 1.44 (t, $3 \mathrm{H}$, $\left.\mathrm{CH}_{3}\right), 2.53\left(\mathrm{~s}, 3 \mathrm{H}, \mathrm{COCH}_{3}\right), 4.45\left(\mathrm{q}, 2 \mathrm{H}, \mathrm{N}-\mathrm{CH}_{2}\right), 6.99-7.34(\mathrm{~m}$, $3 \mathrm{H}, \mathrm{ArH}), 7.50-7.66(\mathrm{~m}, 4 \mathrm{H}, \mathrm{ArH}), 7.96-8.02(\mathrm{~m}, 1 \mathrm{H}, \mathrm{ArH})$, 8.11-8.21 (m, 2H, ArH), 8.66-8.70 (m, $1 \mathrm{H}, \mathrm{Ar}-\mathrm{H}) ;{ }^{13} \mathrm{C} \mathrm{NMR}$ $\left(\mathrm{CDCl}_{3}\right)$ 8/ppm: 14.17, 21.64, 37.98, 109.79, 112.34, 115.63, $116.51,119.98,120.02,121.44,121.89,122.13,123.30$, $125.16,127.71,127.93,129.35,134.49,134.75,139.84$, 142.17, 156.42, MS (m/z): $358(\mathrm{M}+\mathrm{H})^{+}$. HRMS(ESI): calculated for $\mathrm{C}_{23} \mathrm{H}_{19} \mathrm{NNaO}_{3}(\mathrm{M}+\mathrm{Na})$ 380.175794, found 380.175801 .

\section{General Procedure for the Synthesis of Compounds 4(a-e)}

Aryl ester 3 ( $1.0 \mathrm{~g}, 3 \mathrm{mmol}$ ) was dissolved in dry pyridine $(10 \mathrm{~mL})$ and to this reaction mixture powdered potassium hydroxide $(1.65 \mathrm{~g}, 3 \mathrm{mmol})$ was added with constant stirring. The reaction mixture was stirred at room temperature for $3 \mathrm{~h}$. After completion of the reaction (monitored by TLC), the contents were poured over crushed ice and acidified with conc. $\mathrm{HCl}$. The pale yellow colored solid product obtained was filtered and recrystallized from ethanol to get pure compounds 4(a-e), which gave a negative test for ester.

1-(3,5-dichloro-2-hydroxyphenyl)-3-hydroxy-3-(9-methyl9H-carbazol-3-yl)prop-2-en-1-one (4a) Pale yellow colored solid; Yield (73\%); $R_{\mathrm{f}}=0.51$ ( $6 \%$ ethylacetate in $\mathrm{n}$-hexane); m.p. $168-170{ }^{\circ} \mathrm{C}$; IR (KBr) $\tilde{v}_{\max } / \mathrm{cm}^{-1}: 1155(\mathrm{C}-\mathrm{Cl}), 1592$ (C=O), 2976 (enol OH), $3065(\mathrm{OH}) ;{ }^{1} \mathrm{H}$ NMR (DMSO-d $\left.d_{6}\right)$ $\delta / p p m: 1.36$ (t, 3H, $\left.\mathrm{CH}_{3}\right), 4.50$ (q, 2H, N-CH $), 7.25(\mathrm{~m}, 1 \mathrm{H}$, $=\mathrm{CH}$ enol), 7.50-7.57 (m, 2H, ArH), 7.65-7.77 (m, 2H, ArH), 7.82-8.31 (m, 5H, ArH), $12.57(\mathrm{~s}, 1 \mathrm{H}, \mathrm{OH}), 16.89(\mathrm{~s}, 1 \mathrm{H}$, enolic $H$ ); ${ }^{13}$ C NMR (DMSO- $d_{6}$ ) $\delta / p p m: ~ 14.19,37.71,107.19$, $108.84,109.06,110.04,110.70,115.41,120.25,120.48$, $121.00,121.65,122.34,122.53,125.40,126.36,127.26$, 129.13, 134.05, 139.86, 142.19, 145.45, 168.06; MS (m/z): $426(\mathrm{M}+\mathrm{H})^{+}$. HRMS (ESI): calculated for $\mathrm{C}_{23} \mathrm{H}_{17} \mathrm{Cl}_{2} \mathrm{NNaO}_{3}$ $(\mathrm{M}+\mathrm{Na})$ 448.047769, found 448.047534 .
1-(5-chloro-2-hydroxyphenyl)-3-(9-ethyl-9H-carbazol-3yl)-3-hydroxyprop-2-en-1-one (4b) Pale yellow colored solid; Yield (68\%); $R_{\mathrm{f}}=0.55$ ( $6 \%$ ethylacetate in $\mathrm{n}$-hexane); m.p. $137-138{ }^{\circ} \mathrm{C}$; IR (KBr) $\tilde{v}_{\max } / \mathrm{cm}^{-1}: 1131(\mathrm{C}-\mathrm{Cl}), 1591$ (C=O), 2979 (enol OH), $3065(\mathrm{OH}) ;{ }^{1} \mathrm{H}$ NMR (DMSO-d $d_{6}$ ) $\delta / p p m: 1.35\left(\mathrm{t}, 3 \mathrm{H}, \mathrm{CH}_{3}\right), 4.52\left(\mathrm{q}, 2 \mathrm{H}, \mathrm{N}-\mathrm{CH}_{2}\right), 7.32(\mathrm{~m}, 1 \mathrm{H}$, $=\mathrm{CH}$ enol), 7.51-7.58 (m, 2H, ArH), 7.68-7.74 (m, 2H, ArH), $7.80(\mathrm{~m}, 1 \mathrm{H}, \mathrm{ArH}), 8.0(\mathrm{~m}, 1 \mathrm{H}, \mathrm{ArH}), 8.28-8.36(\mathrm{~m}, 3 \mathrm{H}, \mathrm{ArH})$, $9.09(\mathrm{~m}, 1 \mathrm{H}, \mathrm{ArH}), 12.48(\mathrm{~s}, 1 \mathrm{H}, \mathrm{OH}), 16.27$ (bs, $1 \mathrm{H}$, enolic $\mathrm{H}) ;{ }^{13} \mathrm{C}$ NMR (DMSO- $d_{6}$ ) $\delta / \mathrm{ppm}$ : 14.16, 37.70, 109.26, $110.04,110.55,120.15,120.49,121.23,121.57,122.38$, $122.73,122.99,123.34,126.89,127.51,133.49,138.59$, 140.67, 142.54, 159.75, 168.47, 203.07; MS (m/z): 392 $(\mathrm{M}+\mathrm{H})^{+}$. HRMS (ESI): calculated for $\mathrm{C}_{23} \mathrm{H}_{18} \mathrm{CINNaO}_{3}(\mathrm{M}+\mathrm{Na})$ 414.086740, found 414.086855 .

1-(5-bromo-2-hydroxyphenyl)-3-(9-ethyl-9H-carbazol-3yl)-3-hydroxyprop-2-en-1-one (4c)

Pale yellow colored solid; Yield (63\%); $R_{f}=0.52(6 \%$ ethylacetate in n-hexane); m.p. 148-149 ${ }^{\circ} \mathrm{C} ; \mathrm{IR}(\mathrm{KBr})$ $\tilde{v}_{\max } / \mathrm{cm}^{-1}: 1023$ (C-Br), 1594 (C=O), 2975 (enol OH), 3327 $(\mathrm{OH}) ;{ }^{1} \mathrm{H}$ NMR (DMSO- $d_{6}$ ) $\delta / \mathrm{ppm}: 1.35\left(\mathrm{t}, 3 \mathrm{H}, \mathrm{CH}_{3}\right), 4.52$ (q, $\left.2 \mathrm{H}, \mathrm{N}-\mathrm{CH}_{2}\right), 6.98(\mathrm{~m}, 1 \mathrm{H},=\mathrm{CH}$ enol), 7.26-7.54 (m, 3H, ArH), 7.69-8.05 (m, 4H, ArH), 8.23-8.32 (m, 2H, ArH), $9.06(\mathrm{~m}, 1 \mathrm{H}$, $\mathrm{ArH}), 11.73(\mathrm{~s}, 1 \mathrm{H}, \mathrm{OH}), 12.61(\mathrm{~s}, 1 \mathrm{H}$, enolic $\mathrm{H}) ;{ }^{13} \mathrm{C} \mathrm{NMR}$ (DMSO-d $d_{6}$ ) $\delta / p p m: ~ 14.18,37.76,106.30,109.05,109.79$, $110.92,111.90,119.36,119.80,120.99,121.88,123.08$, $124.20,124.95,129.57,133.30,138.22,140.53,141.96$, 147.77, 154.78, 164.32, 177.15; MS $(\mathrm{m} / \mathrm{z}): 436(\mathrm{M}+\mathrm{H})^{+}$. HRMS(ESI): calculated for $\mathrm{C}_{23} \mathrm{H}_{18} \mathrm{BrNNaO}_{3} \quad(\mathrm{M}+\mathrm{Na})$ 458.046015, found 458.047019.

3-(9-ethyl-9H-carbazol-3-yl)-3-hydroxy-1-(2-hydroxy-5methylphenyl)prop-2-en-1-one (4d)

Pale yellow colored solid; Yield $(65 \%) ; R_{\mathrm{f}}=0.49(6 \%$ ethylacetate in $\mathrm{n}$-hexane); m.p. $116-117{ }^{\circ} \mathrm{C} ; \mathrm{IR}(\mathrm{KBr})$ $\tilde{V}_{\max } / \mathrm{cm}^{-1}: 1594(\mathrm{C}=\mathrm{O}), 3056$ (enol OH), $3325(\mathrm{OH}) ;{ }^{1} \mathrm{H}$ NMR

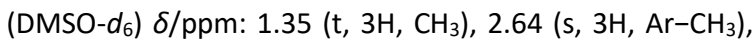
$4.50\left(\mathrm{q}, 2 \mathrm{H}, \mathrm{N}-\mathrm{CH}_{2}\right), 6.96(\mathrm{~m}, 1 \mathrm{H},=\mathrm{CH}$ enol), 7.26-7.29 (m, $1 \mathrm{H}, \mathrm{ArH}), 7.50-7.55(\mathrm{~m}, 2 \mathrm{H}, \mathrm{ArH}), 7.63-7.70(\mathrm{~m}, 3 \mathrm{H}, \mathrm{ArH})$, 7.96-8.29 (m, 3H, ArH), $8.80(\mathrm{~m}, 1 \mathrm{H}, \mathrm{ArH}), 11.73(\mathrm{~s}, 1 \mathrm{H}, \mathrm{OH})$, $12.61(\mathrm{~s}, 1 \mathrm{H}$, enolic $\mathrm{H}) ;{ }^{13} \mathrm{C}$ NMR (DMSO- $d_{6}$ ) $\delta / \mathrm{ppm}: 14.19$, 28.66, 37.54, 108.39, 109.07, 109.59, 110.47, 110.70, $119.80,120.76,121.00,122.86,126.37,127.48,133.08$, $137.77,138.22,139.64,142.18,147.10,150.61,159.40$, 168.06, 203.08; MS $(\mathrm{m} / \mathrm{z}): 372(\mathrm{M}+\mathrm{H})^{+}$. HRMS(ESI): calculated for $\mathrm{C}_{24} \mathrm{H}_{21} \mathrm{NNaO}_{3}(\mathrm{M}+\mathrm{Na})$ 394.012145, found 394.012250.

3-(9-ethyl-9H-carbazol-3-yl)-3-hydroxy-1-(2-hydroxyphenyl)prop-2-en-1-one (4e)

Pale yellow colored solid; Yield (69\%); $R_{f}=0.56(6 \%$ ethylacetate in $n$-hexane); m.p. $134-135{ }^{\circ} \mathrm{C}$; IR (KBr) $\tilde{v}_{\max } / \mathrm{cm}^{-1}: 1677$ (C=O), 3059 (enol OH), $3327(\mathrm{OH}) ;{ }^{1} \mathrm{H} \mathrm{NMR}$

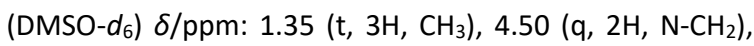
$6.88(\mathrm{~m}, 1 \mathrm{H},=\mathrm{CH}$ enol), 7.27-7.55 (m, 2H, $\mathrm{ArH}), 7.65-7.70$ 
(m, 2H, ArH), 7.80-7.89 (m, 2H, ArH), 8.06-8.32 (m, 2H, ArH), 8.77-8.85 (m, 2H, ArH), $8.97(\mathrm{~m}, 1 \mathrm{H}, \mathrm{ArH}), 11.26(\mathrm{~s}$, $1 \mathrm{H}, \mathrm{OH}$ ), $12.50\left(\mathrm{~s}, 1 \mathrm{H}\right.$, enolic $\mathrm{H}$ ); ${ }^{13} \mathrm{C}$ NMR (DMSO- $d_{6}$ ) $\delta / \mathrm{ppm}$ : $14.19,37.75,109.28,109.58,110.47,111.44,120.47$, $120.99,121.66,121.88,122.11,122.84,123.30,123.98$, $124.95,125.83,126.59,127.26,127.71,128.89,131.96$, 160.36, 168.95; MS $(\mathrm{m} / \mathrm{z}): 358(\mathrm{M}+\mathrm{H})^{+}$. HRMS(ESI): calculated for $\mathrm{C}_{23} \mathrm{H}_{19} \mathrm{NNaO}_{3}(\mathrm{M}+\mathrm{Na})$ 380.175694, found 380.175701 .

\section{General Procedure for the Synthesis of Compounds 5(a-e)}

$\beta$-diketones 4 (0.35g, $1 \mathrm{mmol}$ ) was taken in ethanol (10 mL) and to this reaction mixture hydrazine hydrate $(1.5 \mathrm{~g}, 3$ $\mathrm{mmol}$ ) was added. The reaction mixture was heated under reflux for $3 \mathrm{~h}$. After completion of the reaction (monitored by TLC) the contents were allowed to attain room temperature, then poured into crushed ice and acidified with glacial acetic acid. The brown colored solid product obtained was filtered and recrystallized from ethanol to get pure products $5(\mathrm{a}-\mathrm{e})$.

2,4-dichloro-6-(5-(9-ethyl-9H-carbazol-3-yl)-1H-pyrazol-3yl)phenol (5a)

Brown solid; Yield (72\%); $R_{\mathrm{f}}=0.50$ (7\% ethylacetate in $n$ hexane); m.p. $132-133^{\circ} \mathrm{C}$; IR (KBr) $\tilde{v}_{\max } / \mathrm{cm}^{-1}: 1189(\mathrm{C}-\mathrm{Cl})$, $1455(\mathrm{C}=\mathrm{N}), 3246(\mathrm{NH}), 3373(\mathrm{OH}) ;{ }^{1} \mathrm{H}$ NMR (DMSO- $\left.d_{6}\right)$

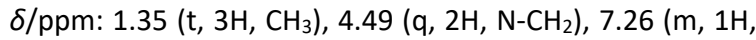
$\mathrm{CH}$ pyrazole), 7.50-7.54 (m, 3H, ArH), 7.66-7.70 (m, 3H, ArH), 7.94-8.29 (m, 3H, ArH), $8.80(\mathrm{~s}, 1 \mathrm{H}, \mathrm{NH}), 12.61(\mathrm{~s}, 1 \mathrm{H}$, $\mathrm{OH}) ;{ }^{13} \mathrm{C}$ NMR (DMSO- $d_{6}$ ) $\delta / \mathrm{ppm}: 13.86,37.79,98.96$, $108.92,109.20,111.12,117.86,118.65,118.94,119.20$, $119.55,120.60,122.58,123.48,126.03,126.32,126.52$, 129.01, 131.82, 140.28, 140.50, 155.24; MS (m/z): 422 $(\mathrm{M}+\mathrm{H})^{+}$. HRMS (ESI): calculated for $\mathrm{C}_{23} \mathrm{H}_{18} \mathrm{Cl}_{2} \mathrm{~N}_{3} \mathrm{O}(\mathrm{M}+\mathrm{H})^{+}$ 422.082144, found 422.082963.

\section{4-chloro-2-(5-(9-ethyl-9H-carbazol-3-yl)-1H-pyrazol-3-yl)} phenol (5b)

Brown solid; Yield (70\%); $R_{\mathrm{f}}=0.52$ (7\% ethylacetate in $n$ hexane); m.p. $178-179{ }^{\circ} \mathrm{C}$; IR (KBr) $\tilde{v}_{\max } / \mathrm{cm}^{-1}: 1026(\mathrm{C}-\mathrm{Cl})$, $1438(\mathrm{C}=\mathrm{N}), 3050(\mathrm{NH}), 3385(\mathrm{OH}) ;{ }^{1} \mathrm{H}$ NMR (DMSO-d $\left.d_{6}\right)$ $\delta / p p m: 1.36\left(\mathrm{t}, 3 \mathrm{H}, \mathrm{CH}_{3}\right), 4.54\left(\mathrm{q}, 2 \mathrm{H}, \mathrm{N}-\mathrm{CH}_{2}\right), 7.23-7.28(\mathrm{~m}$ $1 \mathrm{H}, \mathrm{CH}$ pyrazole), 7.30-7.35 (m, 1H, ArH), 7.50-7.58 (m, 2H, $\mathrm{ArH})$, 7.62-7.70 (m, 1H, ArH), 7.73-7.82 (m, $2 \mathrm{H}, \mathrm{ArH})$, 7.88-8.37 (m, 4H, ArH), 9.08 (s, 1H, NH), $12.46(\mathrm{~s}, 1 \mathrm{H}, \mathrm{OH})$; ${ }^{13} \mathrm{C}$ NMR (DMSO- $d_{6}$ ) $\delta / \mathrm{ppm}: 13.85,37.92,108.08,108.97$, $109.21,113.63,114.32,119.93,120.29,120.67,120.80$, $120.92,121.48,122.64,123.34,124.70,125.77,126.18$, $126.43,127.00,128.50,140.64,142.17,181.64 ; \mathrm{MS}(\mathrm{m} / \mathrm{z})$ : $388(\mathrm{M}+\mathrm{H})^{+}$. HRMS (ESI): calculated for $\mathrm{C}_{23} \mathrm{H}_{19} \mathrm{CIN}_{3} \mathrm{O}(\mathrm{M}+\mathrm{H})^{+}$ 388.121116, found 388.121056.

4-bromo-2-(5-(9-ethyl-9H-carbazol-3-yl)-1H-pyrazol-3-yl) phenol (5c)

Brown solid; Yield (68\%); $R_{\mathrm{f}}=0.48$ (7\% ethylacetate in n-hexane); m.p. $143-144{ }^{\circ} \mathrm{C} ; \quad I R(\mathrm{KBr}) \tilde{v}_{\max } / \mathrm{cm}^{-1}: 1055$ (C-Br), 1451(C=N), $3052(\mathrm{NH}), 3327(\mathrm{OH}) ;{ }^{1} \mathrm{H}$ NMR (DMSO$\left.d_{6}\right) \delta / p p m: 1.36\left(\mathrm{t}, 3 \mathrm{H}, \mathrm{CH}_{3}\right), 4.51\left(\mathrm{q}, 2 \mathrm{H}, \mathrm{N}-\mathrm{CH}_{2}\right), 7.22(\mathrm{~m}$, $1 \mathrm{H}, \mathrm{CH}$ pyrazole), 7.28-7.51 (m, 3H, ArH), 7.53-7.68 (m, 3H, ArH), 7.75-8.34 (m, 4H, ArH), $9.08(\mathrm{~s}, 1 \mathrm{H}, \mathrm{NH}), 12.34(\mathrm{~s}, 1 \mathrm{H}$, $\mathrm{OH}) ;{ }^{13} \mathrm{C}$ NMR (DMSO-d $d_{6}$ ) $\delta / \mathrm{ppm}: 13.74,37.53,98.84$, $108.83,109.79,111.90,117.93,118.15,118.61,119.10$ $119.79,120.25,122.12,123.31,123.77,124.93,125.83$, $129.79,131.42,139.85,140.19,152.08 ; \mathrm{MS}(\mathrm{m} / \mathrm{z}): 432$ $(\mathrm{M}+\mathrm{H})^{+}$. HRMS (ESI): calculated for $\mathrm{C}_{23} \mathrm{H}_{19} \mathrm{BrN}_{3} \mathrm{O}(\mathrm{M}+\mathrm{H})^{+}$ 432.141115, found 432.141156 .

\section{2-(5-(9-ethyl-9H-carbazol-3-yl)-1H-pyrazol-3-yl)-4-} methylphenol (5d)

Brown solid; Yield (71 \%); $R_{f}=0.56$ (7\% ethylacetate in $\mathrm{n}$ hexane); m.p. $123-124{ }^{\circ} \mathrm{C}$; IR ( $\left.\mathrm{KBr}\right) \tilde{v}_{\max } / \mathrm{cm}^{-1}: 1439(\mathrm{C}=\mathrm{N})$, 3054(NH), $3385(\mathrm{OH}) ;{ }^{1} \mathrm{H}$ NMR (DMSO- $\left.d_{6}\right) \delta / \mathrm{ppm}: 1.49(\mathrm{t}$, $\left.3 \mathrm{H}, \mathrm{CH}_{3}\right), 2.55$ (s, 3H, Ar- $\left.\mathrm{CH}_{3}\right), 4.44$ (q, 2H, N-CH $), 6.99(\mathrm{~m}$, $1 \mathrm{H}, \mathrm{CH}$ pyrazole), 7.29-7.45 (m, 3H, ArH), 7.46-7.51 (m, 2H, ArH), 7.53-8.16 (m, 5H, ArH), $10.20(\mathrm{~s}, 1 \mathrm{H}, \mathrm{NH}), 10.91(\mathrm{~s}$, $1 \mathrm{H}, \mathrm{OH}$ ); ${ }^{13} \mathrm{C}$ NMR (DMSO- $d_{6}$ ) $\delta / \mathrm{ppm}: 13.96,28.84,37.98$, $83.18,108.83,109.28,112.54,117.04,118.15,118.60$, $119.79,121.21,122.66,123.30,125.16,127.93,128.45$, $129.57,134.75,139.84,142.85,156.64,168.06,170.81 ; \mathrm{MS}$ $(\mathrm{m} / \mathrm{z}): 368(\mathrm{M}+\mathrm{H})^{+}$. HRMS (ESI): calculated for $\mathrm{C}_{24} \mathrm{H}_{22} \mathrm{~N}_{3} \mathrm{O}$ $(\mathrm{M}+\mathrm{H})^{+}$368.101135, found 368.101179.

2-(5-(9-ethyl-9H-carbazol-3-yl)-1H-pyrazol-3-yl) phenol (5e) Brown solid; Yield (69\%); $R_{\mathrm{f}}=0.49$ (7\% ethylacetate in $\mathrm{n}$ hexane); m.p. $151-152^{\circ} \mathrm{C}$; IR ( $\left.\mathrm{KBr}\right) \tilde{v}_{\max } / \mathrm{cm}^{-1}: 1560(\mathrm{C}=\mathrm{N})$, $3363(\mathrm{NH}), 3676(\mathrm{OH}) ;{ }^{1} \mathrm{H}$ NMR (DMSO- $\left.d_{6}\right) \delta / \mathrm{ppm}: 1.42(\mathrm{t}$, $\left.3 \mathrm{H}, \mathrm{CH}_{3}\right), 4.48\left(\mathrm{q}, 2 \mathrm{H}, \mathrm{N}-\mathrm{CH}_{2}\right), 6.93(\mathrm{~m}, 1 \mathrm{H}, \mathrm{CH}$ pyrazole), $7.05(\mathrm{~m}, 1 \mathrm{H}, \mathrm{ArH}), 7.25-7.33(\mathrm{~m}, 2 \mathrm{H}, \mathrm{ArH}), 7.48-7.65(\mathrm{~m}$, $4 \mathrm{H}, \mathrm{ArH}), 7.94-8.17(\mathrm{~m}, 4 \mathrm{H}, \mathrm{Ar}-\mathrm{H}), 9.11(\mathrm{~s}, 1 \mathrm{H}, \mathrm{NH}), 12.31(\mathrm{~s}$, $1 \mathrm{H}, \mathrm{OH}$ ); ${ }^{13} \mathrm{C}$ NMR (DMSO- $d_{6}$ ) $\delta / \mathrm{ppm}: 13.96,37.76,98.62$, $108.61,109.80,117.26,118.60,119.80,120.99,122.10$, $123.08,123.98,125.61,126.81,127.92,128.22,129.10$, 134.26, 139.41, 142.40, 151.50, 157.31; MS $(\mathrm{m} / \mathrm{z}): 354$ $(\mathrm{M}+\mathrm{H})^{+}$. HRMS (ESI): calculated for $\mathrm{C}_{23} \mathrm{H}_{20} \mathrm{~N}_{3} \mathrm{O}(\mathrm{M}+\mathrm{H})^{+}$ 354.112130, found 354.112190.

Acknowledgment. The Authors are grateful to the Principal of ACS College, Satral and SSGM College, Kopargaon for providing the necessary facilities, SAIF University of Punjab, Chandigarh and Central Instrumentation Facility (CIF) SPPU, Pune for providing the characterization.

Supplementary Information. Supporting information to the paper is attached to the electronic version of the article at: http://doi.org/10.5562/cca3353.

\section{REFERENCES}

[1] J. Bergman, B. Peloman, Pure Appl. Chem. 1990, 62, 1967. 
[2] A. Gluszynska, Eur. J. Med. Chem. 2015, 94, 405.

[3] B. P. Bandgar, L. K. Adsul, S. V. Lonikar, H. V. Chavan, S. N. Shringare, S. A. Patil, S. S. Jalde, B. A. Koti, N. A. Dhole, R. N. Gacche, A. Shirfule, J. Enzyme Inhib. Med. Chem. 2013, 28, 593.

[4] C. K. Ryu, S. Y. Lee, N. Y. Kim, J. A. Hong, J. H. Yoon, A. Kim, Bioorg. Med. Chem. Lett. 2011, 21, 427.

[5] P. Rajakumar, K. Sekar, V. Shanmugaiah, N. Mathivanan, Eur. J. Med. Chem. 2009, 44, 3040.

[6] K. S. Gudmundsson, S. D. Boggs, P. R. Sebahar, L. D. Richardson, A. Spaltenstein, P. Golden, P. B. Sethna, K. W. Brown, K. Moniri, R. Harvey, K. R. Romines, Bioorg. Med. Chem. Lett. 2009, 19, 4110.

[7] Y. Hajbi, C. Neagoie, B. Biannic, A. Chilloux, E. Vedrenne, B. Baldeyrou, C. Bailly, J. Y. Merour, S. Rosca, S. Routier, A. Lansiaux, Eur. J. Med. Chem. 2010, 45, 5428.

[8] F. M. Ho, H. C. Kang, S. T. Lee, Y. Chao, Y. C. Chen, L. J. Huang, W. W. Lin, Biochem. Pharmacol. 2007, 74, 298.

[9] M. J. Thompson, J. C. Louth, S. M. Little, M. P. Jackson, Y. Boursereau, B. N. Chen, I. Coldham, Chem. Med. Chem. 2012, 7, 578.

[10] H. Kaur, S. Kumar, P. Vishwakarma, M. Sharma, K. K. Saxena, A. Kumar, Eur. J. Med. Chem. 2010, 45, 2777.

[11] M. H. Block, S. Boyer, W. Brailsford, D. R. Brittain, D. Carroll, S. Chapman, D. S. Clarke, C. S. Donald, K. M. Foote, L. Godfrey, A. Ladner, P. R. Marsham, D. J. Masters, C. D. Mee, M. R. O’Donovan, J. E. Pease, A. G. Pickup, J. W. Rayner, A. Roberts, P. Schofield, A. Suleman, A. V. Turnbull, J. Med. Chem. 2002, 45, 3509.

[12] N. Haider, R. Jbara, J. Kaferbock, U. Traar, ARKIVOC 2009, (vi), 38.

[13] I. Bennett, N. J. Broom, R. Cassels, J. S. Elder, N. D. Masson, P. J. O'Hanlon, Bioorg. Med. Chem. Lett. 1999, 9, 1847.

[14] G. D. Diana, P. M. Carabateas, R. E. Johnson, G. L. Williams, F. Pancic, J. C. Collins, J. Med. Chem. 1978, 21,889 .
[15] G. D. Crouse, M. J. McGowan, R. J. Boisvenue, J. Med. Chem. 1989, 32, 2148.

[16] T. Nishiyama, S. Shiotsu, H. Tsujita, Polym. Degrad. Stab. 2002, 76, 435.

[17] N. Acton, A. Brossi, D. L. Newton, M. B. Sporn, J. Med. Chem. 1980, 23, 798.

[18] L. Tchertanov, J. F. Mouscadet, J. Med. Chem. 2007, 50, 1133.

[19] T. Huaijun, Z. Zhiguo, C. Changjie, K. Zhang, Russ. J. Org. Chem. 2009, 45, 573.

[20] S. A. H. El-Feky, Z. K. Abd El-Samii, N. A. Osman, J. Lashine, M. A. Kamel, H. K. Thabet, Bioorg. Chem. 2015, 58, 104.

[21] S. Sidique, R. Ardecky, Y. Su, S. Narisawa, N. D. P. Cosford, Bioorg. Med. Chem. Lett. 2009, 19, 222.

[22] J. B. Shi, W. J. Tang, X. B. Qi, R. Li, X. H. Liu, Eur. J. Med. Chem. 2015, 90, 889.

[23] H. V. Chavan, B. P. Bandgar, L. K. Adsul, V. D. Dhakane, P. S. Bhale, V. N. Thakare, V. Masand, Bioorg. Med. Chem. Lett. 2013, 23, 1315.

[24] A. M. Vijesh, A. M. Isloor, S. Prashant, S. Sundershan, H. Kun Fun, Eur. J. Med. Chem. 2013, 62, 410.

[25] F. Zhang, L. Gan, C. He Zhou, Bioorg. Med. Chem. Lett. 2010, 20, 1881.

[26] S. N. Shelke, G. R. Mhaske, S. Gadakh, C. Gill, Bioorg. Med. Chem. Lett. 2010, 24, 7200.

[27] S. N. Shelke, G. R. Mhaske, D. B. Bonifácio Vasco, M. B. Gawande, Bioorg. Med. Chem. Lett. 2012, 17, 5727.

[28] P. Chen, Y. Weng, Li. Niu, Y. Chen, L. Wu, C. Tung, Q. Yang, Angew. Chem. Int. Ed. 2016, 55, 2759.

[29] C. Hauser, F. Swamer, J. Adama, J. Org. React. 1954, 8,168

[30] C. A. Kraus, B. S. Fulton, S. H. Woo, J. Org. Chem. 1984, 49, 3212.

[31] A. P. Keche, G. D. Hatnapure, R. T. Tale, A. H. Rodge, S. S. Birajdar, V. M. Kambale, Med. Chem. Res. 2013, 22, 1480. 